\title{
Relativistic Phenomena in Two-Quantum Annihilation of Electron-Positron Pairs
}

\author{
A. BARANOWSKI* \\ Institute of Experimental Physics, University of Wrocław \\ pl. Maxa Borna 9, 50-204 Wrocław, Poland
}

The kinematics of $\mathrm{e}^{+} \mathrm{e}^{-}$pair and annihilation quanta momentum vectors have been presented basing on ellipse and spheroid properties. Using the formulae resulting from the Lorentz transformation of momentum and energy there was pointed out that the aberration and the longitudinal and transversal Doppler effects are inseparably associated.

PACS numbers: 78.70.Bj, 03.30.+p

\section{Introduction}

Two-quantum annihilation was applied for a long time to the solid state studies. However, there still exist some subtleties, which are not connected with the physics and mathematics of this phenomenon [1-7], but rather with individual thoughts of the momentum vector kinematics, especially in the three-dimensional case. This problem for two-dimensional case has been developed earlier [3-5]. Also in Refs. [2-7] it was pointed out that the longitudinal Doppler effect has essential importance and transversal one is practically not crucial but interesting from the theoretical point of view. It seems, there is also an aberration but it has no importance in practical studies. In Sect. 2 one studies up this phenomenon qualitatively by constructing two- and three-dimensional images of the momentum vectors kinematics. In Sect. 3 we introduce after Ref. [2] several useful formulae based on the Lorentz transformation. This transformation (see Appendix) permits us to calculate all needed features of two-quantum annihilation.

\section{Kinematics of the $\mathrm{e}^{+} \mathrm{e}^{-}$pair and annihilation quanta momentum vectors}

Let us consider the sphere, which is the geometrical place of all $\boldsymbol{p}_{i}$ vectors tips in the rest frame. The radius of this sphere is equal to $p_{i}=m_{0} c$. The

*e-mail: abar@ifd.uni.wroc.pl 


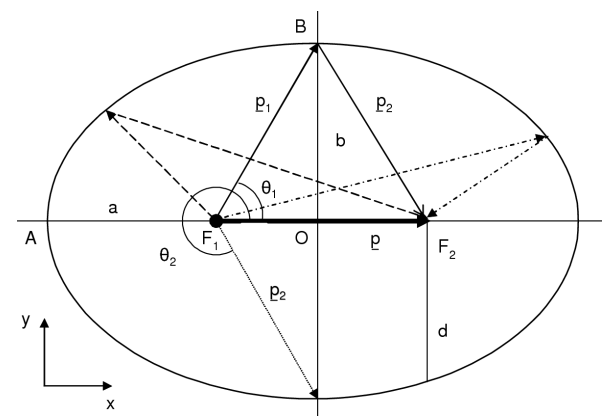

Fig. 1. Geometrical construction pointed up the relationship between quanta and annihilating pair momentum vectors. All symbols are explained in Sect. 2.

problem is how to transform this sphere to the laboratory frame. The answer to this question is spheroid in the laboratory frame. In Fig. 1 one can see the ellipse, which is the geometrical place of all $p_{i}$ with the origin in focus $F_{1}$. Here $a=O A$ is the semi-major axis and $b=O B$ the semi-minor axis of the ellipse, $c=O F_{1}$ and $c=O F_{2}$. The eccentricity is defined as $e=c / a$. The distance from a focus of the ellipse to the ellipse itself, measured along a line perpendicular to the major axis is equal to $d=b^{2} / a$. This formula is a special case of the equation

$$
r=\frac{d}{1+e \cos \theta}
$$

for $\theta=\pi / 2$, where $r$ - distance from a focus to ellipse for any angle $\theta$ between $r$ and the major axis $a$. Now we assign the annihilation parameters to the ellipse ones. Dashed lines indicate two other cases of $p_{i}$ pairs presented as an example and fulfilling the condition $p_{i}+p_{i+1}=2 a$ for all odd $i$.

In the case presented we have

$$
\boldsymbol{p}_{1}+\boldsymbol{p}_{2}=\boldsymbol{p}, \quad p_{1}=p_{2} \text { and } p_{1}+p_{2}=2 a .
$$

The focus $F_{1}$ is the place of pair annihilation, the vector $\boldsymbol{p}$ means the pair momentum. Let us know that $p$ is a distance between foci and therefore equals to $2 c$. The copy of vector $\boldsymbol{p}_{2}$ has been shown by a dotted line in the physically correct place, which is more geometrically suitable. In this construction the revolution symmetry around major axis exists, creating the ellipsoid of revolution or another words - spheroid. Such spheroid is described by Eq. (2.2), however it should be known here that the relations between vector lengths are not realistic because the effect was strongly enhanced in Fig. 1 for more clarity of this picture. Basing on formulae (2.1), (2.2), and the equation of ellipsoid of revolution

$$
\frac{x^{2}}{a^{2}}+\frac{y^{2}}{b^{2}}+\frac{z^{2}}{b^{2}}=1
$$

four exemplary spheroids were constructed and exposed in Fig. 2.

Let us now calculate the parameter $b$. The summary momentum of quanta in the laboratory system is 
a

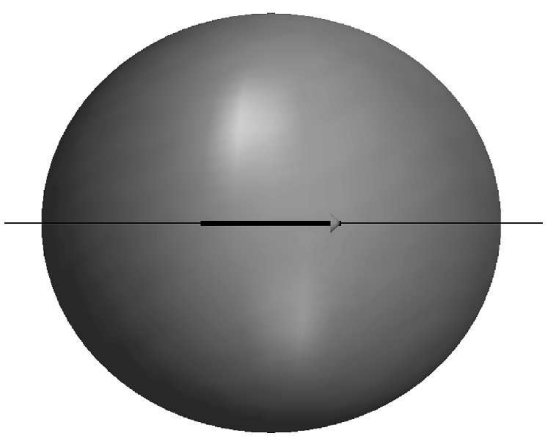

C

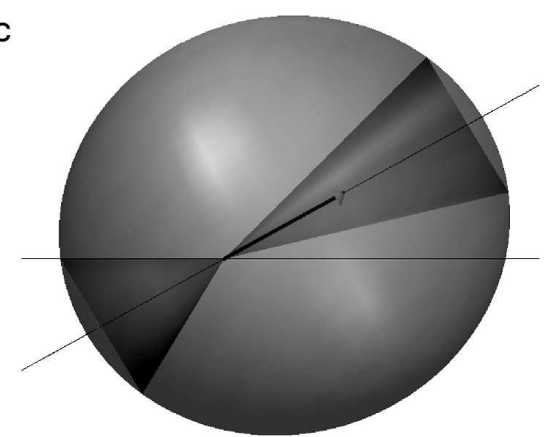

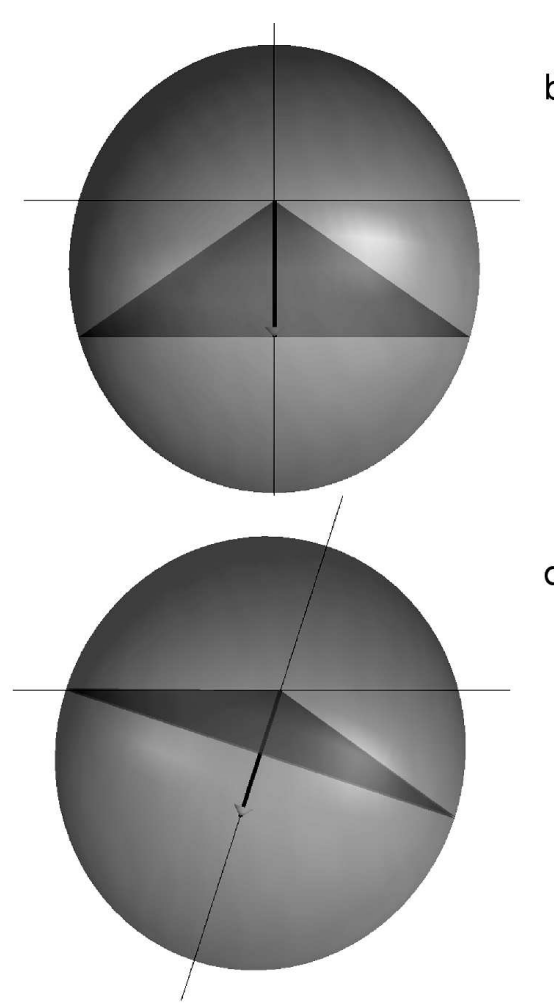

Fig. 2. Three-dimensional construction of the momentum spheroids. Bold vector corresponds to pair momentum. The conical like subfigures inside spheroids indicate three-dimensional distribution of annihilation quanta momentum vectors. Four examples with characteristic different angle between $x$ axis (the horizontal line) and the direction of vector $\boldsymbol{p}$ are presented: (a) $\boldsymbol{p}$ parallel to $x$ axis; (b) $\boldsymbol{p}$ perpendicular to $x$ axis; (d) intermediate $\boldsymbol{p}$ angle for $\theta=\pi / 6$; (c) the case for $\theta^{\prime}=\pi / 2$ in the rest frame.

$$
\begin{aligned}
& \frac{E^{2}}{c^{2}}=4 m_{0}^{2} c^{2}+p^{2}, \\
& a^{2}=p_{1,2}^{2}=m_{0}^{2} c^{4}+p^{4} / 4 \quad \text { and } \quad b=\sqrt{p_{1,2}^{2}-\frac{p^{2}}{4}},
\end{aligned}
$$

so at last one obtains

$$
\begin{aligned}
& b=m_{0} c, \\
& a=m_{0} c \sqrt{1+k^{2} / 4},
\end{aligned}
$$

where $k=p / m_{0} c$.

Taking into account geometrical properties of the ellipse, it can be easy to show that for another interesting case, when $p_{i}$ is perpendicular to the $x$ axis, the result is 


$$
p_{i}=m_{0} c \frac{1}{\sqrt{1+k^{2} / 4}} .
$$

If the direction of $p_{i}$ is parallel to $x$ axis, the momenta $p_{1}$ and $p_{2}$ fulfill the following relation:

$$
\begin{aligned}
& p_{1}=\frac{p}{2}+\sqrt{m_{0}^{2} c^{4}+\frac{p^{2}}{4}}, \\
& p_{2}=\sqrt{m_{0}^{2} c^{4}+\frac{p^{2}}{4}}-\frac{p}{2} .
\end{aligned}
$$

Obviously, we have $p_{1}-p_{2}=p$.

We know that the spheroidal shape of surface of momenta comes from different values of $\theta^{\prime}$ in the rest frame, which moves along the $x$ axis. An observer in the laboratory system can see the spheroid oriented in different directions, when the direction of pair momentum vector changes in space. It is worth knowing that the focus $F_{1}$ is the point of rotation of the spheroid. The observer should register only those events (momenta), which form the conical surface inside the spheroid. Some comments about conical like shapes presented in Fig. 2 should be made. This shape clearly demonstrates that the solid angle corresponding to annihilation $\gamma$ rays moving towards the same hemisphere as annihilating pair is unequal to the angle corresponding to those ones moving towards the opposite hemisphere. Maybe this phenomenon could be called an aberration?

\section{Energy and momentum transformation in relativistic mechanics}

Following [2] and formulae from Appendix one can write

$$
\begin{aligned}
& x^{2}+y^{2}+x^{2}=c^{2} t^{2}, \\
& E^{2}=p^{2} c^{2}+m_{0}^{2} c^{4} .
\end{aligned}
$$

Putting $m_{0}=0$ in (3.2) we obtain the expression (3.3) which allows us to presume the same way of relativistic transformation of momentum components as for the space ones. Also, we expect the energy should transform as a time

$$
\begin{aligned}
& p_{x}^{2}+p_{y}^{2}+p_{z}^{2}=\frac{E^{2}}{c^{2}}, \\
& x^{\prime}=\frac{x-V t}{\sqrt{1-V^{2} / c^{2}}}, \quad t^{\prime}=\frac{t-V x / c^{2}}{\sqrt{1-V^{2} / c^{2}}} .
\end{aligned}
$$

After (3.3) and (3.4), putting $\gamma=\left(1-V^{2} / c^{2}\right)^{-1 / 2}$, we may write the relevant formulae in the following form:

$$
\begin{aligned}
& p_{x}^{\prime}=\gamma\left(p_{x}-V \frac{E}{c^{2}}\right), \quad p_{x}=\gamma\left(p_{x}^{\prime}+V \frac{E^{\prime}}{c^{2}}\right), \\
& p_{y, z}^{\prime}=p_{y, z}, \quad p_{y, z}=p_{y, z}^{\prime}
\end{aligned}
$$

for momentum. Consequently

$$
E^{\prime}=\gamma\left(E-V p_{x}\right), \quad E=\gamma\left(E^{\prime}+V p_{x}^{\prime}\right)
$$

for energy transformation. 
If we take into account momenta and energy of annihilation photons in the center of mass of annihilating pair (primed coordinates), then for any $p^{\prime}$ and $\theta^{\prime}$ associated with $p^{\prime}$ one can write

$$
p_{x}^{\prime}=\frac{h \nu^{\prime}}{c} \cos \theta^{\prime}, \quad p_{y}^{\prime}=\frac{h \nu^{\prime}}{c} \sin \theta^{\prime}, \quad p_{z}^{\prime}=0, \quad E^{\prime}=h \nu^{\prime} .
$$

Making the use of (3.5) we get the following formulae

$$
\begin{aligned}
& p_{x}=\frac{h \nu}{c} \cos \theta=\gamma\left(\frac{h \nu^{\prime}}{c} \cos \theta^{\prime}+V \frac{h \nu^{\prime}}{c^{2}}\right), \\
& p_{y}=\frac{h \nu}{c} \sin \theta=\frac{h \nu^{\prime}}{c} \sin \theta^{\prime}, \\
& p_{z}=0 .
\end{aligned}
$$

For transformation of the energy of annihilation quanta from the rest frame to the laboratory coordinates we get the expression

$$
E=h \nu=\gamma\left(h \nu^{\prime}+V \frac{h \nu^{\prime}}{c} \cos \theta^{\prime}\right) .
$$

The simplification of the terms (3.7) to (3.10) leads to the following formulae:

$$
\begin{aligned}
& \nu \cos \theta=\gamma \nu^{\prime}\left(\cos \theta^{\prime}+\frac{V}{c}\right), \\
& \nu \sin \theta=\nu^{\prime} \sin \theta^{\prime}, \\
& \nu=\gamma \nu^{\prime}\left(1+\frac{V}{c} \cos \theta^{\prime}\right) .
\end{aligned}
$$

Dividing (3.11) by (3.13) one gets a well-known formula joining the relations between $\theta$ and $\theta^{\prime}$ angles in both the laboratory and the rest frame

$$
\cos \theta=\frac{\cos \theta^{\prime}+\frac{V}{c}}{1+\frac{V}{c} \cos \theta^{\prime}} .
$$

Let us consider, basing on (3.14), two cases in the laboratory system. The first, when the annihilation quantum flies perpendicularly to the direction of positronelectron motion and the second one when the directions of both quanta momentum vectors and pair momentum vector are parallel. The first case corresponds to $\theta=\pi / 2$ and, from (3.14), it follows that $\cos \theta^{\prime}=-V / c$. Inserting this to (3.13) one obtains the following:

$$
\nu=\nu^{\prime} \gamma^{-1}
$$

The above equation presents the phenomenon known as the transversal Doppler effect. The case with $\theta=0$ implies $\theta^{\prime}=0$. It leads to the following formula:

$$
\nu_{+}=\nu^{\prime}\left(\frac{1+V / c}{1-V / c}\right)^{1 / 2} .
$$

This is the situation when the annihilating pair moves towards the observer in the laboratory frame. The opposite case for $\theta=\pi$ and $\theta^{\prime}=\pi$ leads to the equation 


$$
\nu_{-}=\nu^{\prime}\left(\frac{1-V / c}{1+V / c}\right)^{1 / 2} .
$$

Expressions (3.16) and (3.17) represent the so-called longitudinal Doppler effect. Let us calculate

$$
\begin{aligned}
& \Delta_{+}=\nu_{+}-\nu^{\prime}, \\
& \Delta_{-}=\nu^{\prime}-\nu_{-}, \\
& \Delta_{+}-\Delta_{-}=2(\gamma-1) \nu^{\prime} .
\end{aligned}
$$

The inequality of $\Delta_{+}$and $\Delta_{-}$is clearly seen, but only for $\nu^{\prime}=m_{0} c^{2} / h$ in $(3.18)$ and (3.19). Modifying $\nu$ in the following manner:

$$
\nu^{\prime \prime}=\nu^{\prime} \gamma
$$

and substituting $\nu^{\prime \prime}$ by $\nu^{\prime}$ to (3.18) and (3.19) one obtains zero on the right hand side of (3.20).

This involves, the point of reference for $\pm \Delta \nu$ should be equal to $p_{i} c / h$, where $p_{i}$ is established by vector $\boldsymbol{p}_{1}$ or $\boldsymbol{p}_{2}$ as marked in Fig. 1 . In this specific state the angle $\theta^{\prime}$ in the rest frame is assumed to be equal to the angle $\pi / 2$ and $\theta_{2}-\theta_{1}-\pi$ (the measure of noncollinearity of vectors $\boldsymbol{p}_{1}$ and $\boldsymbol{p}_{2}$ in Fig. 1) achieves the largest value. Also we have $\pm \Delta \nu=0$. Using Eqs. (3.7) through (3.10) we are able to calculate all possible relations between the angles and momentum vectors in the rest frame and as well as in the laboratory frame.

\section{Summary}

In the present paper some ideas of three-dimensional representation of two-quantum annihilation phenomenon were introduced. It seems the interesting insight on energy and momentum transformation in terms of special relativity explained the Doppler effects from the other point of view [2]. In a recall of Refs. [2, 3]) it has been indicated also the possibility of calculation of various annihilation characteristics, basing on the relations between the parameters of the ellipse being the cross-section in $x y$ plane of the spheroid.

\section{Appendix}

In order to derive the Lorentz transformation equations [1] one assumes the constant value of the light velocity in all inertial systems moving in $x$ direction. Secondly, the primed coordinate system moves along $x$ axis with the velocity $V$ and it is the light source rest frame. For both systems the following equations have to be fulfilled:

$$
\begin{aligned}
& x^{2}+y^{2}+z^{2}=c^{2} t^{2}, \\
& x^{\prime 2}+y^{\prime 2}+z^{\prime 2}=c^{2} t^{\prime 2} .
\end{aligned}
$$

Initially, let us apply the simplest, the Galileo transformation to coordinate $x^{\prime}$ :

$$
x^{\prime}=x-V t, \quad y^{\prime}=y, \quad z^{\prime}=z, \quad t^{\prime}=t .
$$

Inserting (A.3) to (A.2) we have 


$$
\begin{aligned}
& (x-V t)^{2}+y^{2}+z^{2}=c^{2} t^{2}, \\
& x^{2}-2 x V t+V^{2} t^{2}+y^{2}+z^{2}=c^{2} t^{2} .
\end{aligned}
$$

As a result we have obtained expression (A.4), similar to (A.1), but with the additional element (underlined). It means that the transformation should be more complex. In this moment we assume a further transformation of the time $t^{\prime}$ in the form shown below:

$$
\begin{aligned}
& x^{\prime}=x-V t, \quad y^{\prime}=y, \quad z^{\prime}=z, \quad t^{\prime}=t+k x, \\
& \underline{-2 x V t}+V^{2} t^{2}+x^{2}+y^{2}+z^{2}=c^{2} t^{2} \underline{+c^{2} t k x}+c^{2} k^{2} x^{2} .
\end{aligned}
$$

The resultant equation requests for elimination of marked terms with mixed products $x t$

$$
-2 x V t=2 c^{2} t k x \quad \text { hence } \quad k=-\frac{V}{c^{2}} .
$$

Including $k$ to $(\mathrm{A} .5)$ we obtain

$$
\begin{aligned}
& x^{2}-\frac{x^{2} V^{2}}{c^{2}}+y^{2}+z^{2}=c^{2} t^{2}-V^{2} t^{2}, \\
& x^{2}\left(1-\frac{V^{2}}{c^{2}}\right)+y^{2}+z^{2}=c^{2} t^{2}\left(1-\frac{V^{2}}{c^{2}}\right) .
\end{aligned}
$$

After grouping, owing to terms related to $x$ and $t$ coordinates, we can finally write the well-known form of the Lorentz transformation equations, both for primed and unprimed coordinate systems

$$
\begin{aligned}
& x^{\prime}=\frac{x-V t}{\sqrt{1-V^{2} / c^{2}}}, \quad t^{\prime}=\frac{t-V x / c^{2}}{\sqrt{1-V^{2} / c^{2}}}, \\
& x=\frac{x^{\prime}+V t^{\prime}}{\sqrt{1-V^{2} / c^{2}}}, \quad t=\frac{t^{\prime}+V x^{\prime} / c^{2}}{\sqrt{1-V^{2} / c^{2}}} .
\end{aligned}
$$

\section{Acknowledgments}

This work was supported by the internal grant of the University of Wrocław No. 2016/IFD/06.

\section{References}

[1] C. Kittel, W.D. Knight, M.A. Ruderman, Mechanics, Berkeley Physics Course, PWN, Warszawa 1967 (in Polish).

[2] C. Katz, An Introduction to the Special Theory of Relativity, PWN, Warszawa 1967 (in Polish).

[3] W. Świątkowski, Ph.D. thesis, University of Wrocław, Wrocław 1966.

[4] W. Świątkowski, in: Proc. the 33rd Polish Seminar on Positron Annihilation, Turawa (Poland) 2001, Ed. K. Jerie, University of Opole, University of Wrocław, Opole 2001.

[5] A.M. Baldin, W.I. Goldanskij, W.M. Maksimienko, I.L. Rozental, Kinematics of Nuclear Reaction, Atomizdat, Moskwa 1968 (in Russian). 
[6] J. Dryzek, Introduction to Positron Annihilation Spectroscopy, Jagiellonian University, Kraków 1997 (in Polish).

[7] R. Eisberg, R. Resnick, Quantum Physics of Atoms, Molecules, Solids, Nuclei and Particles, PWN, Warszawa 1983 (in Polish). 